\title{
Calvarial Bone Implantation and in vivo Imaging of Tumor Cells in Mice
}

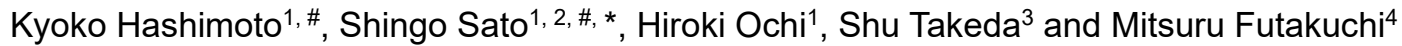

\author{
1Department of Physiology and Cell Biology, Tokyo Medical and Dental University (TMDU), Graduate \\ School, Tokyo 113-8519, Japan; ${ }^{2}$ Department of Orthopaedic Surgery, Tokyo Medical and Dental \\ University (TMDU), Graduate School, Tokyo 113-8519, Japan; ${ }^{3}$ Division of Endocrinology, Toranomon \\ Hospital Endocrine Center, Tokyo 105-8470, Japan; ${ }^{4}$ Department of Pathology, Nagasaki University \\ Graduate School of Biomedical Sciences, Nagasaki, 852-8523, Japan \\ *For correspondence: satoshin.phy2@tmd.ac.jp \\ \#Contributed equally to this work
}

\begin{abstract}
[Abstract] Bone is one of common metastasis sites for many types of cancer. In bone metastatic microenvironment, tumor-bone interactions play a significant role in the regulation of osteolytic or osteoblastic bone metastasis. In order to investigate the direct interaction between tumor cells and bone tissue, it is essential to generate appropriate animal models that mimic the behavior of tumor cells in bone metastatic lesions. Calvarial implantation model (bone invasion model) is a newly-established animal model that accurately recapitulates the behavior of tumor cells in the tumor-bone microenvironment. The surgical technique for tumor cell implantation is simpler than intracardiac, intraarterial, or intraosseous injection techniques. This model can be useful for the identification of key factors driving tumor-induced osteolytic or osteoblastic changes.
\end{abstract}

Keywords: Tumor microenvironment, Tumor-bone interaction, Tumor cell implantation, Calvaria implantation model, Osteolytic bone metastasis, Osteoblastic bone metastasis

[Background] Bone is one of frequent metastasis sites for many types of cancer, including prostate, breast, and lung cancer. Based on their radiographic appearance, bone metastatic lesions are mainly classified into two types: osteolytic or osteoblastic lesions. These lesions are induced by an imbalance between bone formation and bone resorption in the tumor-bone microenvironment. In bone metastatic lesions, tumor cells interact with many types of cells, including osteoblasts, osteoclasts, or mesenchymal stem cells. According to previous reports, the increased bone-resorbing or bone-forming activity is mediated by many paracrine factors secreted by metastasized tumor cells. However, the detailed mechanisms responsible for the osteolytic or osteoblastic phenotype remain to be fully elucidated. To investigate the molecular mechanisms underlying how metastasized tumor cells can induce osteolytic or osteoblastic phenotypes, it is essential to generate animal models that mimic the behavior of tumor cells in bone metastatic lesions.

Calvarial implantation model (bone invasion model) was originally established by Mitsuru Futakuchi and his colleagues [Lynch et al., 2005]. This model accurately recapitulates the behavior of various tumor cells (e.g., rat prostate cancer cells [Lynch et al., 2005], murine breast cancer cells [Wilson et al., 2008], etc.) in bone metastatic microenvironment. In addition, surgical technique required for the 
implantation of tumor cells is simpler than intracardiac, intra-arterial or intraosseous injection techniques. On the other hand, this model has a couple of limitations. It does not represent the metastasis process from primary organs to bone. Besides, calvaria is not a typical metastasis site for most of cancers.

This model is especially useful in identifying key factors driving tumor-induced osteolytic or osteoblastic changes. Using this model, Futakuchi and his colleagues has demonstrated an important role of MMP-7, cathepsin G, or transforming growth factor $\beta$ (TGF $\beta$ ) signaling in tumor-induced osteolysis or tumor growth in bone metastatic microenvironment (Lynch et al., 2005; Sato et al., 2008; Wilson et al., 2008; Futakuchi et al., 2009). In another study, the effect of bisphosphonate or RANKLtargeting therapy was evaluated using this model (Hikosaka et al., 2006; Futakuchi et al., 2018). Hashimoto et al. also used this model to investigate the tumor-stromal interactions by cancer-secreted microRNAs (Hashimoto et al., 2018).

\section{Materials and Reagents}

1. Pipette tips

2. $1 \mathrm{ml}$ tuberculin syringe (TERUMO, catalog number: SS-01T)

3. $21 \mathrm{G}$ needle (TERUMO, catalog number: NN-2138R)

4. $23 \mathrm{G}$ needle (TERUMO, catalog number: NN-2325R)

5. $15 \mathrm{ml}$ or $50 \mathrm{ml}$ tube

6. Immunodeficient mice (8-10-week-old BALB/c nu/nu or NOD-SCID mice)

7. Tumor cells (e.g., 4T1, Cl66, and Cl66M2 murine breast cancer cells [Wilson et al., 2008], rat prostate cancer cells [Lynch et al., 2005], MDA-MB-231-Luc human breast cancer cells [Cell Biolabs, catalog number: AKR-231], etc.)

Note: Luciferase stably expressing tumor cells (e.g., MDA-MB-231-Luc cells) were used for IVIS in vivo imaging system.

8. Anesthetic agents

A combination anesthetic was prepared with $0.3 \mathrm{mg} / \mathrm{kg}$ of medetomidine (ZENOAQ), $4.0 \mathrm{mg} / \mathrm{kg}$ of midazolam (Sandoz K.K.), and $5.0 \mathrm{mg} / \mathrm{kg}$ of butorphanol (Meiji Seika Pharma Co., Ltd.)

9. $\mathrm{PBS}$

10. Matrigel Matrix Basement (Corning, catalog number: 354234 , storage temperature $-20^{\circ} \mathrm{C}$ )

11. D-luciferin (Summit Pharmaceuticals International, catalog number: XFL-1, storage temperature $\left.-20^{\circ} \mathrm{C}\right)$

12. $2.5 \mathrm{~g} / \mathrm{L}$ Trypsin/1 mmol/L EDTA solution (NACALAI TESQUE, catalog number: $32777-15$ )

\section{Equipment}

1. Pipettes

2. Centrifuge (TOMY, EX-125)

3. IVIS In Vivo Imaging System (PerkinElmer, IVIS Lumina III) 
4. Micro-computed tomography (Scan: Rigaku, R_mCT2; Analysis: Ratoc, TRI/FCS-BON)

\section{Procedure}

A. Tumor cell preparation

1. Culture tumor cells according to the indicated culture method.

Note: 4T1, Cl66, and Cl66M2 murine breast cancer cells, MDA-MB-231-Luc human breast cancer cells, and rat prostate cancer cells were used in the author's previous studies.

2. Aspirate the culture media and add PBS to rinse the cells.

3. Aspirate PBS and add $0.25 \%$ trypsin/EDTA solution.

Note: Ensure the solution covers the entire surface of the cells by tilting the culture plate.

4. Incubate the cells at $37{ }^{\circ} \mathrm{C}$ for a few minutes in a humidified $5 \% \mathrm{CO}_{2}$ environment.

5. Add the culture media to neutralize the trypsin, detach the adherent cells by pipetting the media back and forth, and make a single cell suspension.

6. Transfer the cell suspension to a $15 \mathrm{ml}$ or $50 \mathrm{ml}$ tube and centrifuge it at $500 \times \mathrm{g}$ for $5 \mathrm{~min}$ at $4{ }^{\circ} \mathrm{C}$.

7. Aspirate the supernatant, resuspend the cell pellet in cold PBS, and count the cell number.

8. Centrifuge the cell suspension again and resuspend the cell pellet with cold Matrigel at $3 \times 10^{6}$ cells per $100 \mu$ l Matrigel.

Note: Matrigel is a liquid at $4{ }^{\circ} \mathrm{C}$. It polymerizes into a solid gel when maintained at $24-37^{\circ} \mathrm{C}$.

9. Keep the resuspended cells on ice until the cells are implanted.

B. Tumor cell implantation on mouse calvarial bone (Figure 2)

\section{Anatomy of mouse calvarial bone}

Mouse calvarial bone is mainly composed of frontal, parietal and interparietal bones (Figures $1 \mathrm{~A}$ and $1 \mathrm{~B}$ ). There are two major midline sutures: interfrontal suture between the paired frontal bones and sagittal suture between the paired parietal bones. In addition, there are two major transverse sutures: coronal sutures between the frontal and parietal bones and lambdoid sutures between the parietal and interparietal bones (Figure 1A). 
A

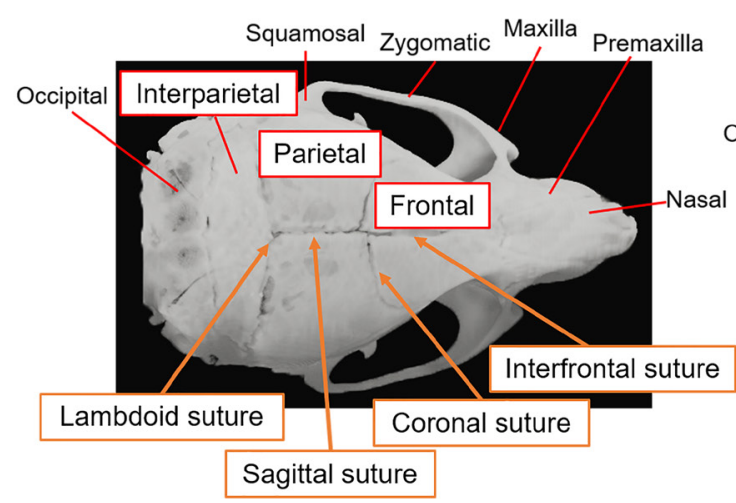

B

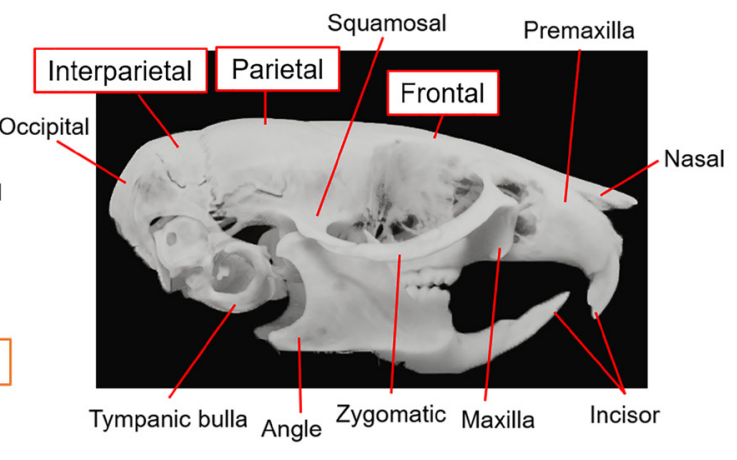

Figure 1. Three-dimensional $\mu C T$ reconstructions of mouse calvarial bone. A. Top view. $B$. Lateral view.

1. Prepare 8-10-week-old BALB/c-nu/nu or NOD-SCID mice for the experiment.

2. Anesthetize a mouse by intraperitoneal injection of anesthetic agents as previously reported (Kawai et al., 2011).

3. Insert a $21 \mathrm{G}$ needle into the forehead skin (Figure $2 \mathrm{~A}, \mathrm{X}$ : Needle insertion point) and scratch the periosteum of calvarial bone with the tip of the needle in order to expose the surface of bone (Figure 2B).

Note: Complete exposure of bone surface is important for tumor implantation.

4. Transfer the cell suspension into a $1 \mathrm{ml}$ syringe and attach a $23 \mathrm{G}$ needle.

Note: Ensure the cells are homogeneously suspended by pipetting up and down before the transfer.

5. Insert the injection needle from the same skin hole and inject $100 \mu \mathrm{l}\left(3 \times 10^{6}\right.$ cells $)$ of the cell suspension over the calvarial bone (Figure $2 \mathrm{C}$ ).

6. After pulling the needle out, pinch the injection hole with fingers for about $30 \mathrm{sec}$ until Matrigel polymerizes into a solid gel (Figures 2D and 2E).

Note: Take care not to let the cell suspension leak through the hole.

7. Place the injected mouse on a heating pad and monitor it until it completely becomes awake. 
A

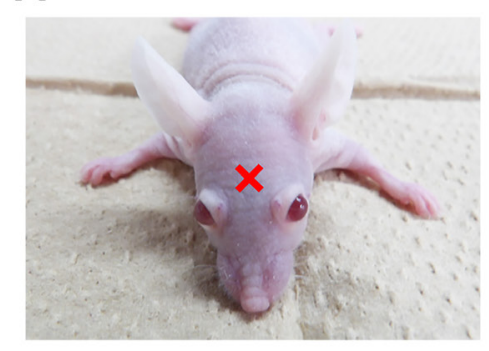

D

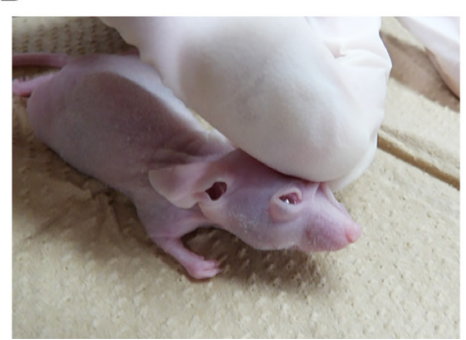

B

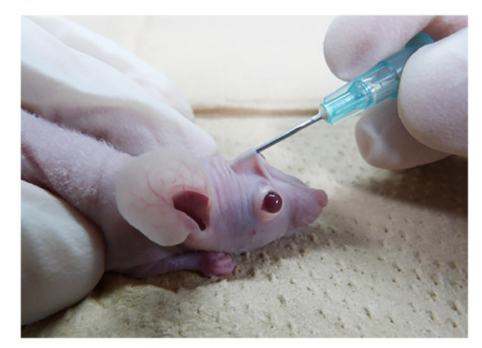

E

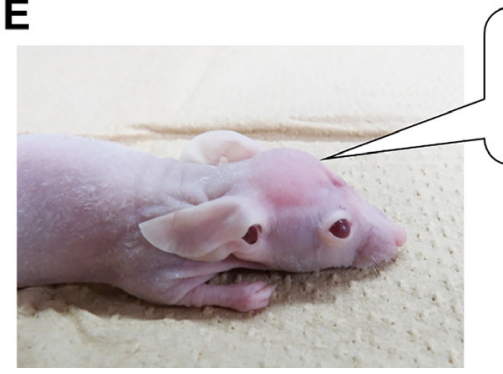

C
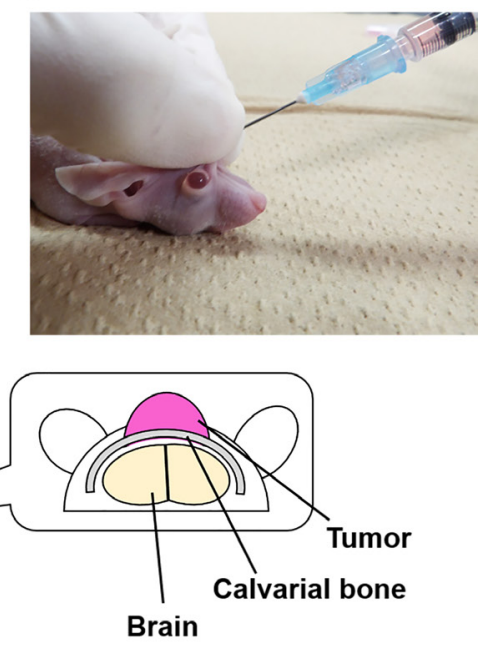

Figure 2. Cancer cell implantation on mouse calvarial bone

C. After implantation

1. Monitor tumor growth regularly.

2. Measure tumor size at the implanted site weekly with a vernier caliper.

3. Perform IVIS in vivo imaging and micro-computed tomography $(\mu \mathrm{CT})$ scanning weekly or every 2 weeks and check the size of tumor, osteolytic lesions or osteoblastic lesions (see Data analysis).

4. Sacrifice the implanted mouse at approximately 3-6 weeks (based on $\mu \mathrm{CT}$ analysis) after the implantation and harvest the resulting tumor for further analyses (e.g., histological analysis and gene expression analysis).

Note: At early time points, little or no change of the calvarial bone is observed. Osteolytic or osteoblastic lesions were visually identified at approximately 2-4 weeks (depend on implanted tumor cells) after the tumor cells are implanted.

\section{Data analysis}

\section{Imaging tumor growth and bone lesions}

1. IVIS imaging system: Luciferin is a common bioluminescent reporter used for in vivo imaging of the expression of luciferase. IVIS imaging system is recommended to be performed weekly after the implantation of luciferase stably expressing tumor cells (e.g., MDA-MB-231-Luc cells, etc.). Before the imaging, $300 \mu \mathrm{l}(4.5 \mathrm{mg})$ of D-luciferin $(15 \mathrm{mg} / \mathrm{ml})$ is injected intraperitoneally into an implanted mouse under anesthetic condition. Images are obtained 10 min after the injection using IVIS Lumina III. The same mouse was investigated at different time points. If the tumor cells are successfully implanted, the bioluminescent intensity is increased gradually after the implantation (Figures 3A and 3B). Sometimes, implanted tumor cells (e.g., LNCaP and C4-2 
human prostate cancer cells, etc.) fail to survive. The precise reason for the failure still remains unclear. Generally, the more cells that were implanted, the more cells tend to survive.

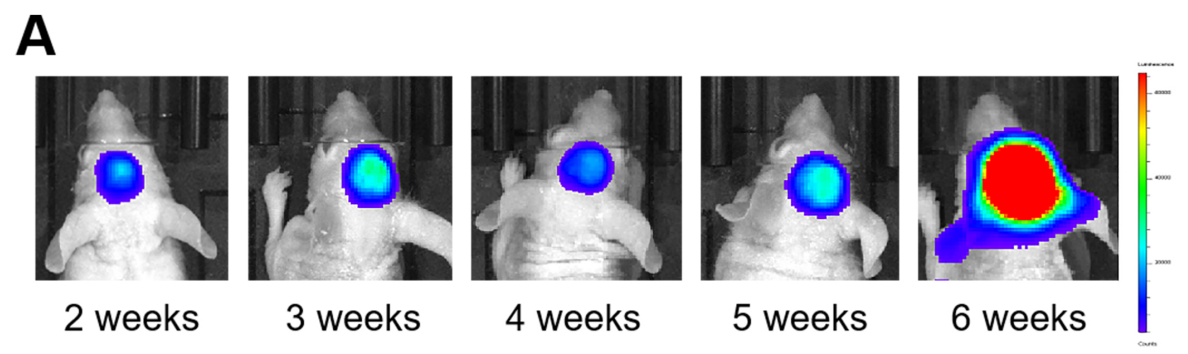

\section{B}

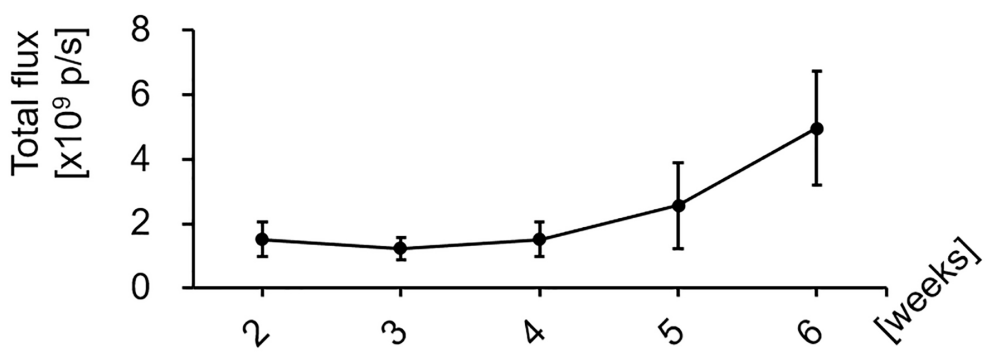

Figure 3. IVIS in vivo imaging of implanted MDA-MB-231-Luc cells. A. IVIS images. B. Bioluminescent intensity of photons (Total flux $[\mathrm{p} / \mathrm{s}]$ ) emitted from the implanted tumor cells. The data are the means \pm SEMs $(n=5)$.

2. Micro-computed tomography $(\mu \mathrm{CT})$ analysis: $\mu \mathrm{CT}$ analysis is recommended to be performed weekly or every 2 weeks after the tumor cells are implanted. $\mu \mathrm{CT}$ scanning was conducted under anesthetic condition using R_mCT2 (Rigaku) with an isometric resolution of $40 \mu \mathrm{m}$, and the images were reconstructed using TRI/FCS-BON (Ratoc). In the case that the osteolytic phenotype-inducing tumor cells (e.g., MDA-MB-231 cells) are implanted, osteolytic lesions can be observed at 4-6 weeks after the implantation. Bone absorption areas become larger gradually (Figure 4A). On the other hand, in the case that the osteoblastic phenotype-inducing tumor cells (e.g., rat prostate cancer cells [Lynch et al., 2005], hsa-miR-940-expressing MDAMB-231 breast cancer cells [Hashimoto et al., 2018], etc.) are implanted, osteoblastic lesions can appear at 10-14 days after the implantation (Figure 4B). 
A

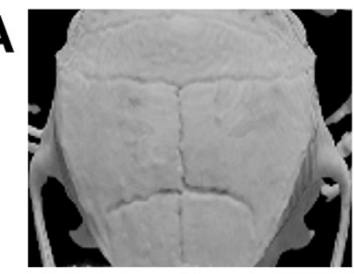

2 weeks

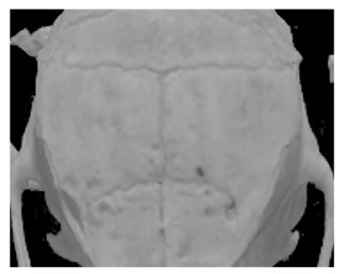

4 weeks

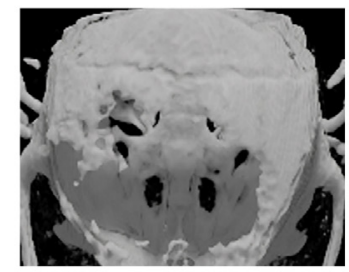

6 weeks

B

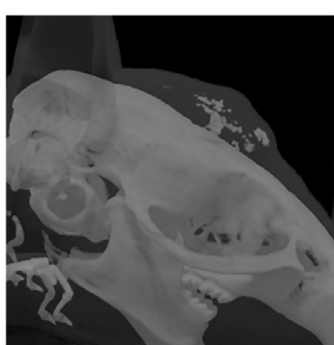

2 weeks

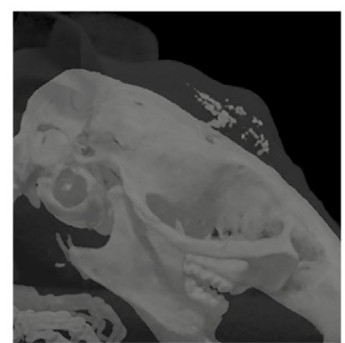

4 weeks

Figure 4. Three-dimensional $\mu \mathrm{CT}$ reconstructions of the implanted site. A. Osteolytic lesions. B. Osteoblastic lesions.

\section{Acknowledgments}

This work was supported by Grants-in-Aid for Scientific Research (KAKENHI, \#24791567 and \#26893068). This work was also supported by the Core Research for Evolutional Science and Technology (CREST, \#JP17gm0610008).

\section{Competing interests}

All authors declare no conflicts of interest.

\section{Ethics}

All animal experiments regarding calvarial bone implantation were performed with approval from the Animal Study Committee of University of Nebraska Medical Center, Nagoya City University, Nagasaki University, and Tokyo Medical and Dental University and conformed to their relevant guidelines and legislation.

\section{References}

1. Futakuchi, M., Nannuru, K. C., Varney, M. L., Sadanandam, A., Nakao, K., Asai, K., Shirai, T., Sato, S. Y. and Singh, R. K. (2009). Transforming growth factor- $\beta$ signaling at the tumor-bone interface promotes mammary tumor growth and osteoclast activation. Cancer Sci 100(1): 7181. 
2. Futakuchi, M., Nitanda, T., Ando, S., Matsumoto, H., Yoshimoto, E., Fukamachi, K. and Suzui, M. (2018). Therapeutic and preventive effects of osteoclastogenesis inhibitory factor on osteolysis, proliferation of mammary tumor cell and induction of cancer stem cells in the bone microenvironment. Int J Mol Sci 19(3): E888.

3. Hashimoto, K., Ochi, H., Sunamura, S., Kosaka, N., Mabuchi, Y., Fukuda, T., Yao, K., Kanda, H., Ae, K., Okawa, A., Akazawa, C., Ochiya, T., Futakuchi, M., Takeda, S. and Sato, S. (2018). Cancer-secreted hsa-miR-940 induces an osteoblastic phenotype in the bone metastatic microenvironment via targeting ARHGAP1 and FAM134A. Proc Natl Acad Sci U S A 115(9): 2204-2209.

4. Hikosaka, A., Futakuchi, M., Ogiso, T., Suzuki, S., Kohri, K. and Shirai, T. (2006). Lack of prophylactic effect of incadronate on skeletal lesions associated with implants of prostate cancer. Eur Urol 49(1): 176-182.

5. Kawai, S., Takagi, Y., Kaneko, S. and Kurosawa, T. (2011). Effect of three types of mixed anesthetic agents alternate to ketamine in mice. Exp Anim 60(5): 481-487.

6. Lynch, C. C., Hikosaka, A., Acuff, H. B., Martin, M. D., Kawai, N., Singh, R. K., Vargo-Gogola, T. C., Begtrup, J. L., Peterson, T. E., Fingleton, B., Shirai, T., Matrisian, L. M. and Futakuchi, M. (2005). MMP-7 promotes prostate cancer-induced osteolysis via the solubilization of RANKL. Cancer Cell 7(5): 485-496.

7. Sato, S., Futakuchi, M., Ogawa, K., Asamoto, M., Nakao, K., Asai, K. and Shirai, T. (2008). Transforming growth factor $\beta$ derived from bone matrix promotes cell proliferation of prostate cancer and osteoclast activation-associated osteolysis in the bone microenvironment. Cancer Sci 99(2): 316-323.

8. Wilson, T. J., Nannuru, K. C., Futakuchi, M., Sadanandam, A. and Singh, R. K. (2008). Cathepsin $G$ enhances mammary tumor-induced osteolysis by generating soluble receptor activator of nuclear factor-kB ligand. Cancer Res 68(14): 5803-5811. 